\section{Autopercepción del estado de salud, las desigualdades en salud y la crisis económica: el caso de España}

\author{
Self-rated health status, health \\ inequalities and economic crisis: \\ the case of Spain
}

Rita Barradas Barata'

${ }^{1}$ Doctora en Medicina Preventiva. Profesora asociada de Epidemiología, Escuela de Ciencias Médicas, Santa Casa de São Paulo. São Paulo, Brasil. \ (iD

doi: 10.18294/sc.2018.1984

Discusión sobre: Spijker J, Gumà J. El efecto de la crisis económica sobre la salud en España según el nivel educativo y la relación con la actividad: ¿importa también la duración de la crisis? Salud Colectiva. 2018;14(4):655670. doi: 10.18294/sc.2018.1297

Hay numerosos artículos que abordan los efectos de la crisis económica que comenzó en 2008 sobre la salud de las poblaciones europeas y estadounidenses, especialmente, en países como España, Portugal y Grecia, que sufrieron los mayores impactos del declive en crecimiento económico y las tasas más altas de desempleo, y adoptaron además medidas de austeridad con recortes significativos en el financiamiento de las políticas sociales ${ }^{(1,2,3)}$.

Sin embargo, los resultados de muchos de los artículos parecen entrar en conflicto entre sí, y algunos han alcanzado conclusiones totalmente opuestas a las que se esperarían con base en el conocimiento actual sobre los determinantes macrosociales y su impacto en la salud(4).

Aunque las relaciones y las mediaciones entre los fenómenos macrosociales -como las crisis económicas- y sus efectos sobre la salud de la población no son fáciles de desenredar adecuadamente, varios autores han utilizado métodos y conceptos apropiados para evitar conclusiones apresuradas y demasiado simplistas. Este es el caso del artículo en discusión ${ }^{(5)}$, en el cual los autores utilizaron una metodología apropiada y analizaron e interpretaron sus hallazgos cuidadosamente.

Una de las ventajas de este artículo, por encima de muchos otros, es que los autores analizaron el estado de salud en 2006, 2010 y 2014 , es decir, antes y durante la crisis económica, lo cual probablemente permita una mejor evaluación de los efectos, teniendo en cuenta la brecha que existe entre el deterioro de las condiciones económicas y la pérdida de efectividad de las políticas sociales como amortiguadoras de los impactos negativos ${ }^{(4,6)}$.

Tanto las políticas sociales del Estado de bienestar, como los mecanismos de solidaridad social, en tanto factores significativos para frenar los efectos dañinos del deterioro del estado económico de las familias, suelen perder su efectividad durante el inicio y la profundización de la crisis hasta la recuperación económica, por las políticas de austeridad impuestas por organismos internacionales o el gobierno nacional, o por la pérdida de la red de solidaridad dada la extensión de la crisis.

Se puede dividir las crisis en distintas fases. La primera oleada es el impacto económico inicial con un descenso en el crecimiento del producto bruto interno y la reducción de trabajos e ingresos familiares, entre otras cuestiones. La segunda oleada está marcada por el impacto social, con un incremento de la tasa de desempleo, una creciente dificultad de los jóvenes para introducirse en el mercado laboral, y las políticas de austeridad que reducen los beneficios sociales. Finalmente, la tercera fase consiste en la recuperación, con una reanudación del crecimiento que puede tener tasas diferentes según país o región. ${ }^{(7)}$

La oportunidad de analizar los efectos hasta 2014 y, por lo tanto, durante un periodo más largo, permitió a los autores observar procesos que no se pudieron ver en estudios previos cuyos análisis incluyeron datos solo de tres o cuatro años después del inicio de la crisis. Otro punto de relevancia 
del artículo es que los autores consideraron en sus análisis e interpretación de resultados las especificidades de la formación social de España y aspectos del escenario político, extremadamente importantes para modular el efecto de la crisis.

Varios autores han tenido un abordaje un tanto ingenuo ya que parecen esperar una relación determinística entre la crisis económica y la salud de la población, sin reconocer que los determinantes macro no son exactamente iguales en diferentes estructuras sociales y que los mecanismos a través de los cuales operan pueden diferir de un escenario a otro. Como fenómeno complejo que afecta no solo la disponibilidad de bienes, sino que también cambia los patrones de los comportamientos relacionados con la salud, la crisis económica puede impactar sobre el estado de salud a través de mecanismos y procesos que den resultados opuestos ${ }^{(2,6,8)}$.

Como en cualquier estudio que intenta analizar la relación entre determinantes sociales y salud, es extremadamente importante elegir tanto indicadores del proceso social como indicadores de salud. Las variables socioeconómicas elegidas por Spijker y Gumá(5) tienen características interesantes para los análisis propuestos. Además de ser una variable fácil de obtener, con pocos valores desconocidos en encuestas poblacionales, el nivel de educación tiene la ventaja de ser estable en poblaciones adultas. Generalmente, para los 30 años ya se ha completado la escolaridad y, por lo tanto, no se espera que varíe en el tiempo para el individuo. Sin embargo, para poblaciones, la distribución del nivel de educación refleja el efecto de cohorte generacional, dado que los niveles de educación suelen aumentar en el tiempo, y reflejan mejorías en las condiciones de vida. Los individuos que en 2006 tenían de 30 a 59 años nacieron entre 1947 y 1976; los que tenían esa edad en 2010, nacieron entre 1951 y 1980; y los que esa edad en 2014, nacieron entre 1955 y 1984 y, por lo tanto, experimentaron distintas oportunidades escolares. Los datos presentados en la Tabla 1 del artículo en discusión ${ }^{(5)}$ muestran exactamente esta tendencia de desplazamiento de la distribución poblacional a niveles más altos durante el periodo de estudio, lo que en sí debería resultar en mejorías en la salud autopercibida.

Las otras tres variables seleccionadas están más sujetas a cambios en el escenario, por lo que pueden reflejar los efectos de la crisis económica más rápidamente. La estructura familiar, la capacidad económica de la familia para enfrentar sus necesidades y el estado de empleo de la familia hacen referencia a aspectos objetivos, que permiten identificar grupos sociales con diferentes vulnerabilidades, cómo pueden ser afectados por la crisis y con qué recursos cuentan para enfrentarla.

La elección de la salud autopercibida, como variable dependiente, es interesante, ya que refleja una evaluación global del estado de salud, que remite al concepto de salud como bienestar y no solo ausencia de enfermedad ${ }^{(9)}$. Los autores que han elegido la tasa de mortalidad o indicadores como esperanza de vida (dependiendo del perfil de mortalidad específica por edad) como variable dependiente no han sido capaces de demostrar el efecto de la crisis sobre la salud, probablemente, porque ambos son indicadores globales que se obtienen al distribuir las muertes por grupo de edad y, por lo tanto, sujetos a múltiples factores relacionados con la estructura de edad, las causas y los determinantes de las muertes y el desempeño de los servicios de salud ${ }^{(8)}$. La salud mental, por ejemplo, es un componente importante del estado de salud, pero no se ve reflejado adecuadamente en los datos de mortalidad o esperanza de vida, salvo por la incidencia de suicidio. Hay evidencia de que durante una crisis empeora el estado mental y aumenta el uso de ansiolíticos ${ }^{(2,10)}$.

Es importante subrayar el aspecto más crucial para entender la paradoja aparente entre la mejora del estado de salud y la crisis económica: los datos globales enmascaran las diferencias entre grupos sociales y en diferentes momentos de la crisis $^{(1,6,8,9,11,12,13)}$.

Aunque Barroso et al. ${ }^{(11)}$ reconocieron, por ejemplo, que entre 2006 y 2012 las muestras estudiadas difieren respecto a determinantes sociales de la salud importantes, 
como nivel de educación (con una reducción en la proporción de individuos con poca o nula escolaridad), lo que favorecería una mejor percepción del estado de salud, afirmaron categóricamente que, a pesar de la crisis, hubo mejoras en el nivel de salud observada.

Los cambios en la distribución de los determinantes de salud antes y durante la crisis son responsables de los distintos procesos, con distintos impactos sobre cada grupo social. De esta manera, la tendencia temporal a largo plazo de mejora del nivel de escolaridad de la población española, no modificada inmediatamente por la crisis, en combinación con la reducción proporcional de individuos sin escolaridad, o con escolaridad básica a favor de estratos con educación secundaria o más, fue definitivamente uno de los elementos que contribuyó directa e indirectamente en la mejoría del estado de salud.

Además de mejor escolaridad, el periodo atestiguó mayor desigualdad de ingresos, traducida en el aumento en el índice de Gini y la redistribución del poder de compra de las familias, con mayor impacto en la población de jóvenes y con preservación relativa de los ingresos de los mayores, gracias a que las pensiones se mantuvieron durante el periodo de crisis. Los ingresos aumentaron en el quintil más alto de distribución y se redujeron en el quintil más bajo. Estos movimientos, en parte positivos y en parte negativos, producen impactos diferentes según la vulnerabilidad de un grupo social pero, en general, tienden a generar mejor salud al afectar desproporcionadamente a los grupos más saludables (como las personas jóvenes) ${ }^{(1,8,12)}$.

Hasta el desempleo puede tener impactos distintos sobre los grupos que perdieron sus trabajos tempranamente en la crisis y los que crónicamente están afuera del mercado. Una porción significativa del desempleo ocurrió en el sector de construcción civil, que emplea personas jóvenes con poca escolaridad y sin calificaciones profesionales. El desempleo, en este grupo, puede haber tenido un efecto paradojal sobre el estado de salud, ya que con la preservación del seguro de desempleo durante los primeros dos años y la reducción de los riesgos asociados a la ocupación en sí, el estado de salud efectivamente podría haber sido percibido como mejor. Asimismo, los recortes en los gastos de salud pública se concentraron más en el deterioro de los salarios del personal de salud que en la oferta de servicios y, por lo tanto, podrían no haber tenido un efecto inmediato en el estado de salud ${ }^{(13)}$.

Como muestran los autores en el artículo discutido $^{(5)}$, la extensión de una crisis puede erosionar progresivamente los mecanismos de compensación, produciendo efectos dañinos en los grupos más vulnerables, así como en las personas cuyo estado económico ha sido profundamente afectado.

\section{REFERENCIAS BIBLIOGRÁFICAS}

1. Coveney M, García-Gómez P, Van Doorslaer E, Van Ourti T. Health disparities by income in Spain before and after the economic crisis. Health Economics.2016;25(Suppl 2):141-158

2. Córdoba-Doña JA, Escolar-Pujolar A, San Sebastián M, Gustafsson PE. How are the employed and unemployed affected by economic crisis in Spain?: educational inequalities, life conditions and mental health in the context of high unemployment. BMC Public Health. 2016;16:267. doi: 10.1186/s12889-016-2934-z.

3. Alvarez-Galvez J, Salinas-Perez JA, Rodero-Cosano ML, Salvador-Carulla L. Methodological barriers to studying the association between the economic crisis and suicide in Spain. BMC Public Health. 2017;17(1):694. doi: 10.1186/s12889-017-4702-0.

4. Tapia Granados JA. La crisis y la salud en España y en Europa: ¿está aumentando la mortalidad? Salud Colectiva. 2014;10(1):81-91.

5. Spijker J, Gumà J. The effect of the economic crisis on health in Spain according to educational level and employment status: Does the duration of the crisis also matter? Salud Colectiva. 2018;14(4):655-670. doi: 10.18294/ sc. 2018.1297.

6. Fernández SC, Ajuria AF, González MPLA, Martín JJM. Diferencias por sexo de la salud percibida antes y durante la crisis económica (2007 y 2011). Revista Española de Salud Pública. 2017;91:201702019.

7. Rajmil L, Siddiqi A, Taylor-Robinson D, Spencer N. Understanding the impact of the economic crisis on child health: the case of Spain. International Journal of Equity in Health. 2015;14:95. doi: 10.1186/s12939-015-0236-1.

8. Arroyo E, Renart G, Saez M. How the economic recession has changed the likelihood of reporting poor selfrated health in Spain. International Journal of Equity in Health. 2015;14:149. doi: 10.1186/s12939-015-0285-5. 
9. Borrell C, Rodríguez-Sanz M, Bartoll X, Malmusi D, Novoa AM. El sufrimiento de la población en la crisis económica del Estado español. Salud Colectiva. 2014:10(1):95-98

10. Pérez-Romero S, Gascón-Cánovas J, Cruz-Sánchez E, Sánchez-Ruiz JF, Parra-Hidalgo P, Monteagudo-Piqueras O. Recesión económica (2006-2012) y cambios en el estado de salud de la población española. Salud Pública de México. 2016;58(1):41-48.

11. Barroso C, Abásolo I, Cáceres JJ. Health inequalities by socioeconomic characteristics in Spain: the economic crisis effect. International Journal of Equity in Health. 2016;15:62. doi: 10.1186/s12939-016-0346-4.

12. Bartoll X, Toffolutti $V$, Malmusi $D$, Palència L, Borrell C, Suhrcke M. Health and health behaviours before and during the Great Recession, overall and by socioeconomic status, using data from four repeated cross-sectional health surveys in Spain (2001-2012). BMC Public Health. 2015;15:865. doi: 10.1186/s12889-015-2204-5.

13. Lostao L, Geyer S, Albaladejo R, Moreno-Lostao A Santos JM, Regidor E. Socioeconomic position and health services use in Germany and Spain during the Great Recession. PLoS One. 2017;12(8):e0183325. doi: 10.1371/ journal.pone.0183325.

\section{FORMA DE CITAR}

Barata RB. Autopercepción del estado de salud, las desigualdades en salud y la crisis económica: el caso de España. Salud Colectiva. 2018;14(4):671-674. doi: $10.18294 /$ sc. 2018.1984 .

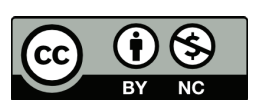

Esta obra está bajo una Licencia Creative Commons Atribución 4.0 Internacional.

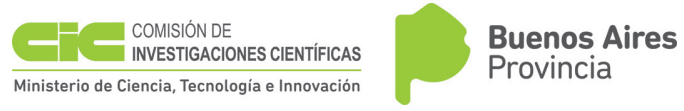

Este artículo fue traducido al español por Vanessa Di Cecco con fondos del Subsidio para Publicaciones de Divulgación Científica (Acta N N 1459/17) de la Comisión de Investigaciones Científicas de la provincia de Buenos Aires.

http://dx.doi.org/10.18294/sc.2018.1984 